\title{
Invasive procedure
}

Sian Cousins, Natalie S Blencowe, Jane M Blazeby

\section{Source}

Sian Cousins, Natalie S Blencowe, Jane M Blazeby. (2019). What is an invasive procedure?

A definition to inform study design, evidence synthesis and research tracking. BMJ Open, vol. 9 (7), e028576.

An invasive procedure is one where purposeful/deliberate access to the body is gained via an incision, percutaneous puncture, where instrumentation is used in addition to the puncture needle, or instrumentation via a natural orifice. It begins when entry to the body is gained and ends when the instrument is removed, and/or the skin is closed. Invasive procedures are performed by trained healthcare professionals using instruments, which include, but are not limited to, endoscopes, catheters, scalpels, scissors, devices and tubes.

Where invasive procedures also involve the administration of a medicinal product, these could be categorised as being part of an 'invasive procedure' when operator skill is required for its administration within the body, that is, when an internal action is performed to administer the product or the product is administered to a targeted anatomical area, for example, Zhu et al. ${ }^{1}$ There are also procedures which involve operator skill to target something inside the body (eg, electromagnetic radiation in the eye) without an incision, percutaneous puncture or instrumentation via a natural orifice. These types of procedures do not fall within the definition of an invasive procedure.

1. Jianhong Zhu, Liangfu Zhou, Feng Ge XingWu. (2006). Tracking Neural Stem Cells in Patients with Brain Trauma. N EngIJ Med, vol. 355 (22), 2376-2378. 